\title{
Independence and chromatic densities of graphs
}

\author{
Anthony Bonato, Jason I. Brown, \\ Graeme Kemkes and Pawę Prąat
}

\begin{abstract}
We consider graph densities in countably infinite graphs. The independence density of a finite graph $G$ of order $n$ is its proportion of independent sets to all subsets of vertices, while the chromatic density is its proportion of proper $n$-colourings to all mappings from vertices of $G$ to $\{1,2, \ldots, n\}$. For both densities, we extend their definition to countable graphs via limits of chains of finite graphs. We show that independence densities exist for all chains, and are unique regardless of which limiting chain is used. We prove that independence densities are always rational; in fact, we prove the stronger fact that the closure of the set of possible values is contained in the rationals. In contrast, we show that the infinite random graph contains chains realizing all real numbers in $[0,1]$ as a chromatic density.
\end{abstract}

KEYWORDS AND PHRASES: Infinite graph, independent sets, graph colouring, graph density, infinite random graph.

\section{Introduction}

An approach to generalizing certain finite graph parameters to infinite graphs is to consider graph densities. Broadly speaking, graph densities are normalized ratios of certain graph parameters. A well-known example is the edge density, or proportion of edges in a graph to all two-element sets of vertices. For an infinite graph, define its upper density as the infimum of all reals $\alpha$ such that the finite subgraphs with edge density greater than $\alpha$ have bounded order. As a corollary of the Erdös-Stone theorem from extremal graph theory (see, for example, [9]), the upper density of a graph is a superparticular number; that is, a number in the set

$$
\{1-1 / n: n \geq 1\} \cup\{1\} \text {. }
$$


Other graph densities include homomorphism density [13], cop density [3] and limiting survival rates in firefighter games $[4,16]$.

A countably infinite graph $G$ may be expressed as the limit of a chain of finite graphs. Suppose we are given a sequence of finite induced subgraphs $\mathcal{C}=\left(G_{n}: n \geq 0\right)$ of $G$ with the properties that $G_{n}$ is an induced subgraph of $G_{n+1}$, and

$$
V(G)=\bigcup_{n=0}^{\infty} V\left(G_{n}\right) .
$$

We write $\lim _{n \rightarrow \infty} G_{n}=G$, and say that $G$ is the limit of the chain $\mathcal{C}$. As we will see, limits are powerful tools for proving density results for infinite graphs.

For a non-negative parameter $\rho$ defined for finite graphs, it is natural to extend the definition of $\rho$ to countable graphs by continuity:

$$
\rho\left(\lim _{n \rightarrow \infty} G_{n}\right)=\lim _{n \rightarrow \infty} \rho\left(G_{n}\right) .
$$

If the limit graph is a finite graph, then the chain is eventually constant, and the limit exists. However, in the infinite case, limits may either depend on the chain used, fail to exist, or be infinite. We only consider cases where the limit exists; further, we consider parameters where $\rho\left(G_{n}\right)$ is bounded. As with edge densities, we first normalize the parameter $\rho$ (in some way) so that its value is in the interval $[0,1]$.

We consider two examples of such parameters in the present work: the independence and chromatic densities. The independence density of a finite graph $G$ of order $n$ is its proportion of independent sets to all subsets of vertices, while the chromatic density is its proportion of proper $n$-colourings to all mappings from $V(G)$ to $\{1,2, \ldots, n\}$. We show in Theorem 2.2 that independence densities exist for all chains, and that independence densities are unique regardless of which limiting chain is used. We prove in Theorem 2.4 the surprising result that independence densities are always rational; in fact, we prove a stronger result that the closure of the set of possible values is contained in the rationals. In contrast to these results the main result of Section 3 is Theorem 3.1, where we show that by choosing different chains the infinite random graph realizes all real numbers in $[0,1]$ as a chromatic density.

Throughout, all graphs we consider are undirected, simple, and countable (that is, either finite or countably infinite). As we only discuss countably infinite sets, we refer to the cardinality of an infinite set by $\infty$. 


\section{Independence density}

Let $I(G)$ denote the set of independent sets of a graph $G$ (including the empty set), and let $i(G)=|I(G)|$. Several authors have studied the number of independent sets in graphs arising from various families (see, for example, $[11,12,14,18])$. The parameter $i(G)$ is sometimes called the Fibonacci number of a graph [15], since $i\left(P_{n}\right)=F(n+2)$, where $P_{n}$ is the path with $n$ vertices and $F(m)$ is the $m$ th Fibonacci number. We note in passing that $i(G)$ is the evaluation of the independence polynomial of $G$ at the value 1; see $[6,7,8]$ for more on the independence polynomial. If $G$ is a graph of order $n$, then we have the tight bounds

$$
n+1 \leq i(G) \leq 2^{n} .
$$

As is well-known (see [15]), if $G$ is a spanning subgraph of $H$, then

$$
i(H) \leq i(G)
$$

and if $G \cup H$ is the disjoint union of $G$ and $H$, then

$$
i(G \cup H)=i(G) i(H) .
$$

We define the independence density of graph $G$ of order $n$ to be

$$
\operatorname{id}(G)=\frac{i(G)}{2^{n}}
$$

Note that $\operatorname{id}(G)$ is a rational in $(0,1]$. We may view $\operatorname{id}(G)$ as the probability of choosing an independent set (uniformly at random) from all subsets of $V(G)$. The following lemma follows from (2.1) and (2.2).

Lemma 2.1. Let $G$ and $H$ be finite graphs.

(1) If $G$ is a subgraph of $H$, then $\operatorname{id}(H) \leq \operatorname{id}(G)$.

(2) If $G$ and $H$ are vertex disjoint graphs, then $\operatorname{id}(G \cup H)=\operatorname{id}(G) \operatorname{id}(H)$.

Given a chain $\mathcal{C}=\left(G_{n}: n \geq 0\right)$ whose limit is $G$, we define the independence density of $G$ relative to $\mathcal{C}$ by

$$
\operatorname{id}(G, \mathcal{C})=\lim _{n \rightarrow \infty} \operatorname{id}\left(G_{n}\right)
$$

(assuming the limit exists, which we will show in Theorem 2.2). An immediate but important consequence of Lemma 2.1 is that independence densities relative to chains always exist and are independent of the chain used. 
Theorem 2.2. Let $G$ be a countable graph.

(1) For all chains $\mathcal{C}$ with limit $G, \mathrm{id}(\mathrm{G}, \mathcal{C})$ exists.

(2) Let $\mathcal{C}=\left(G_{n}: n \geq 0\right)$ and $\mathcal{C}^{\prime}=\left(H_{n}: n \geq 0\right)$ be two chains with the same limit graph $G$. Then

$$
\operatorname{id}(G, \mathcal{C})=\operatorname{id}\left(\mathrm{G}, \mathcal{C}^{\prime}\right)
$$

Proof. Item (1) follows from Lemma 2.1(1), as the independence densities of a chain form a non-increasing, bounded set in $[0,1]$. For $(2)$, fix $\varepsilon>0$ a real number. Let $I=\operatorname{id}(G, \mathcal{C})$ and $I^{\prime}=\operatorname{id}\left(G, \mathcal{C}^{\prime}\right)$. There is an $n \geq 0$ such that $\operatorname{id}\left(G_{n}\right) \leq I+\varepsilon$. We have that $G_{n}$ is an induced subgraph of $H_{k}$ for some $k \geq 0$. This implies that

$$
I^{\prime} \leq \operatorname{id}\left(H_{k}\right) \leq \operatorname{id}\left(G_{n}\right) \leq I+\varepsilon
$$

By symmetry, $I \leq I^{\prime}+\varepsilon$.

Theorem 2.2 simplifies the theory of independence densities considerably, since we may work with any chain and arrive at the same limit. Hence, we drop reference to chains, and simply refer to the independence density of $G$, written $\operatorname{id}(G)$.

The independence densities of the infinite complete and empty graphs are 0 and 1, respectively. For a ray (that is, a one-way infinite path), the independence density is 0 as $F(n+2) / 2^{n}$ goes to zero. The infinite star $K_{1, \infty}$ is the limit of the chain of finite stars $K_{1, n}$, which has independence density

$$
\left(2^{n}+1\right) / 2^{n+1}=1 / 2+2^{-n-1}
$$

Hence, $\operatorname{id}\left(K_{1, \infty}\right)=1 / 2$.

We emphasize that independence densities are insensitive to adding independent sets to the graph. Further, graphs which appear considerably sparser than each other can have the same independence density: for this, consider a countably infinite complete graph and the graph formed by taking the disjoint union of countably many edges.

Nevertheless, we think independence densities have interesting properties, as our next result demonstrates. Our main result considers the possible values for independence densities. In a non-empty finite graph $G$, by Lemma 2.1(1) we have that

$$
\operatorname{id}(G) \leq \operatorname{id}\left(K_{2}\right)=\frac{3}{4}
$$


so there are no real numbers in $(3 / 4,1)$ that are independence densities. Other such gaps exist by considering graphs with at most $n$ edges, where $n \geq 2$.

Define

$$
S=\{\operatorname{id}(G): G \text { is a countable graph }\},
$$

and

$$
S^{\prime}=\{\operatorname{id}(G): G \text { is a finite graph }\} .
$$

Then $S^{\prime} \subseteq S, 0 \in S \backslash S^{\prime}$, and by Lemma 2.1 and the continuity of limits, $S$ forms a monoid with 0 with multiplication defined by

$$
\operatorname{id}(G) \operatorname{id}(H)=\operatorname{id}(G \cup H) .
$$

The closures (in the usual topology of $\mathbb{R}$ ) of $S$ and $S^{\prime}$, written $\bar{S}$ and $\overline{S^{\prime}}$, respectively are equal. (To see this, suppose that $G$ is an infinite graph. Then for all $\varepsilon>0$ there is a finite induced subgraph $H$ of $G$ with $|\operatorname{id}(H)-\operatorname{id}(G)|<$ в.)

A graph $G$ containing an infinite matching has $\operatorname{id}(G)=0$. In fact, the size of a maximum matching plays an important (albeit unexpected) role for independence densities. The matching number of $G$, written $\mu(G)$ (also sometimes called $\alpha_{1}(G)$ ), is the cardinality of a maximum matching in $G$. If there is an infinite matching in $G$, then we write $\mu(G)=\infty$.

Theorem 2.3. For a countable graph $G$, if $\operatorname{id}(G)>0$, then $\mu(G)<\infty$, and

$$
(\mu(G)+1) 2^{-2 \mu(G)} \leq \operatorname{id}(G) \leq\left(\frac{3}{4}\right)^{\mu(G)} .
$$

In particular, $\operatorname{id}(G)=0$ if and only if $\mu(G)=\infty$.

Proof. The upper bound follows by noting that by Lemma 2.1(1) that id $(G)$ is maximized in the case $G$ consists of a disjoint union of $\mu(G)$ edges. For the lower bound, define $A$ to be the set of $2 \mu(G)=2 m$ vertices in a fixed maximum matching $M$ with $m$ edges, and $B=V(G) \backslash A$. Observe that $B$ is an independent set. Let $\left(G_{n}: n \geq 0\right)$ be a chain with $G_{0}$ as the subgraph induced by $A$, and suppose that $G_{n}$ has $N$ vertices. Considering vertices in $B \cap V\left(G_{n}\right)$ supplies $\left(2^{N-2 m}\right)$-many independent sets. For each edge of our fixed matching, we show that there are $\left(2^{N-2 m}\right)$-many additional independent sets. This will give us $(m+1) 2^{N-2 \mu(G)}$ independent sets in total, proving the lower bound. 
Fix an edge $e=u v$ of $M$. If $e$ has an endpoint with no neighbours in $B$, then that endpoint can be combined with any subset of $B$, forming $\left(2^{N-2 m}\right)$-many independent sets. Otherwise, both $u$ and $v$ have at least one neighbour in $B$. If the endpoints had different neighbours, then a larger matching could be formed, so the endpoints must have a unique common neighbour $w$ and no other neighbours. We can form an independent set by combining either endpoint with any subset of $B \backslash\{w\}$, supplying the desired $2^{N-2 m}$ independent sets.

Our main result of this section is the following theorem, which shows that the closure of the set of independence densities is contained in the rationals.

Theorem 2.4. For any fixed irrational $q$, there exists $\varepsilon=\varepsilon(q)>0$ such that $|q-x| \geq \varepsilon$ for all $x \in S$, and so $\bar{S} \subseteq \mathbb{Q} \cap[0,1]$.

The following corollary follows immediately from Theorem 2.4.

Corollary 2.5. The independence density of a graph is rational.

Before we move to the proof of Theorem 2.4, we need one more lemma. For a finite graph $G$, define $F(G)$ to be the family of graphs (not necessarily finite) consisting of $G$ and an independent countable set of vertices (which may be joined to vertices in $G$ ). A graph $H \in F(G)$ is called a $G$-flower or flower. We first show the following key observation that independence densities of $G$-flowers are bounded away from any irrational number.

Lemma 2.6. For any irrational $q \in(0,1)$ and any finite graph $G$, there exists $\varepsilon=\varepsilon(q, G)>0$ such that $|q-\mathrm{id}(H)| \geq \varepsilon$ for all $H \in F(G)$.

Proof. Fix a graph $H \in F(G)$. For each subset $S$ of $V(G)$, let $x_{S}$ be the number of vertices in $V(H) \backslash V(G)$ that are joined to every vertex of $S$ and no vertex in $V(G) \backslash S$. Hence, we partition the set of vertices outside of $G$ into $\left(2^{|V(G)|}\right)$-many classes, with membership in a class determined by their adjacency to vertices of $G$. Thus, we may represent $H \in F(G)$ by the vector $\overline{x_{H}}$ with $\left(2^{|V(G)|}\right)$-many coordinates. (For example, $K_{1, n} \in F\left(K_{1}\right)$ has a representation of $(n, 0)$.) The number of independent sets of $H$ is

$$
\sum_{I \in I(G)} 2^{\sum_{\{S \subseteq V(G): S \cap I=\emptyset\}} x_{S}} .
$$

Hence,

$$
\operatorname{id}(H)=\frac{\sum_{I \in I(G)} 2^{-\sum_{\{S \subseteq V(G): S \cap I \neq \emptyset\}} x_{S}}}{2^{|V(G)|}} .
$$


If $x_{S}=\infty$ (that is, in the case there are infinitely many vertices joined to $S$ and no vertex outside $S$ ) then we remove all terms in the (outermost) sum involving $x_{S}$.

For $H$ with vector $\overline{x_{H}}$ for which $\operatorname{id}(H)>q$, then we have two possible situations.

(1) One coordinate of $\overline{x_{H}}$ may be increased and the resulting independence density is at least $q$.

(2) No coordinate can be increased (perhaps some of them are already $\infty$ ) without the resulting independence density becoming smaller than $q$. We call such vectors saturated.

Dually, if we have a vector $\overline{x_{H}}$ for which $\operatorname{id}(H)$ is smaller than $q$, we can have two possible situations.

(1) Some finite coordinate may be decreased (perhaps to 0) and the resulting independence density is at most $q$.

(2) Decreasing any finite coordinate yields an independence density greater than $q$. We call such vectors minimal.

In the case that $\operatorname{id}(H)>q$ where its corresponding vector is not saturated, we can increase the coordinates of $\overline{x_{H}}$, keeping the independence density above $q$, until we reach a graph corresponding to a saturated vector (this vector may have all coordinates equal to $\infty$, but is still saturated by definition). Since for any saturated or minimal vector we obtain a positive rational value for $\operatorname{id}(H)$, it is enough to show that the number of such vectors is finite. If this is indeed the case, then we may define

$$
\varepsilon(q, G)=\min \{|q-\mathrm{id}(H)|\}>0,
$$

where the minimum is taken over all graphs that can be represented as a saturated or minimal vector. (If the number of such vectors is not finite, then the minimum becomes an infimum and it is possible that $\varepsilon(q, G)=0$, which implies that we can get as close to $q$ as we want.)

We now show there are only finitely many saturated or minimal vectors. We first consider saturated vectors. Since there are a finite number of possibilities to place infinities in any saturated vector (exactly $2^{2^{|V(G)|}}$ ), we may fix these positions arbitrarily in advance, and show that the number of vectors of this type is finite. For a contradiction, suppose that for some configuration of infinities, there is an infinite number of saturated vectors. We need to focus on finite coordinates only, so let us rearrange coordinates 
of our saturated vectors as follows: vector $\overline{V_{k}}$ has the following form:

$$
\overline{V_{k}}=\left(x_{k}^{1}, x_{k}^{2}, \ldots, x_{k}^{l}, \infty, \infty, \ldots, \infty\right)
$$

where $x_{k}^{i}$ are finite nonnegative integers. Since we have an infinite family of vectors, there must be at least one coordinate $x_{k}^{i}$ (for some $1 \leq i \leq l$ ) that is not bounded. Without loss of generality, we can assume that there is a sequence of vectors $\left(\overline{V_{k}^{1}}\right)_{k=1}^{\infty}$ such that $x_{k+1}^{1}>x_{k}^{1}$ for $k \geq 1$. Since we restrict ourselves to saturated vectors, no two vectors can be different on the first coordinate only. This implies that there are infinitely many different configurations $\left(x_{k}^{2}, x_{k}^{3}, \ldots, x_{k}^{l}\right)$ that we encounter in the sequence $\left(\overline{V_{k}^{1}}\right)_{k=1}^{\infty}$, so at least one more coordinate $x_{k}^{i}$ is not bounded (for some $2 \leq i \leq l$ ). Without loss of generality, we suppose that $x_{k}^{2}$ is not bounded and so there exists a subsequence $\left(\overline{V_{k}^{2}}\right)_{k=1}^{\infty}$ of $\left(\overline{V_{k}^{1}}\right)_{k=1}^{\infty}$ such that $x_{k+1}^{i}>x_{k}^{i}$ for $k \geq 1$ and $i \in\{1,2\}$. Using the fact that no saturated vector can be obtained by increasing some coordinates of some other saturated vector, we may continue constructing subsequences to show the existence of a sequence of saturated vectors $\left(\overline{V_{k}^{l}}\right)_{k=1}^{\infty}$ such that $x_{k+1}^{i}>x_{k}^{i}$ for $k \geq 1$ and $i \in\{1,2, \ldots, l\}$. As $\overline{V_{2}^{l}}$ is saturated it follows that $\overline{V_{1}^{l}}$ is not (one cannot increase $l$ coordinates of $\overline{V_{1}^{l}}$ to get another saturated vector), which gives a contradiction.

The proof that for a given $q$ and $G$, there are finitely many minimal vectors is symmetric and thus, is omitted. (For that, one can use the symmetry that no minimal vector can be obtained by decreasing some coordinates of some other minimal vector.)

Proof of Theorem 2.4. Fix an irrational $q \in[0,1]$. It follows from Lemma 2.6 that for every finite graph $G$, there exists $\varepsilon=\varepsilon(q, G)>0$ such that $\mid q-$ $\operatorname{id}(H) \mid \geq \varepsilon$ for any $H \in F(G)$. However, there might be a sequence of graphs $\left(G_{i}\right)_{i \in \mathbb{N}}$ such that $\varepsilon\left(q, G_{i}\right)$ tends to zero as $i$ goes to infinity. We will show that this is impossible which finishes the proof of the theorem.

Suppose $G$ is a graph with $\operatorname{id}(G)$ close to $q$; without loss of generality, assume that $\operatorname{id}(G)>0$. Recall that by Theorem $2.3, \operatorname{id}(G) \leq(3 / 4)^{\mu(G)}$. Hence, if we want to be close to $q$, we cannot have $\mu(G) \geq k_{q}$, where

$$
k_{q}=\left\lceil\frac{\log q}{\log (3 / 4)}\right\rceil+1
$$

Therefore, to be close to $q$ we need to have some graph $G$ of order at most $2 k_{q}$ as a subgraph and all other vertices are attached to $G$. (If there is a 
vertex with distance at least two from $G$, then $G$ has a larger matching. Similarly, if there is another connected component, we can increase the size of the matching. Isolated vertices in $G$ may be removed without changing the independence density.) Since we have a finite number of graphs on at most $2 k_{q}$ vertices, we define

$$
\varepsilon(q)=\min \{\varepsilon(q, G)\}
$$

where the minimum is taken over all $G$ with at most $2 k_{q}$ vertices.

An interesting problem is whether $S-S^{\prime}=\{0\}$. One approach to this problem is to classify the independence number of countable graphs with a given finite matching number. We completed this classification in the case $\mu(G)$ is 1 or 2 ; in each case, the independence numbers for all countable graphs correspond to those for finite graphs. For example, if $\mu(G)=1$, then

$$
\operatorname{id}(G) \in\left\{2^{-1}+2^{-A}: A \geq 2\right\} \cup\left\{2^{-1}\right\} .
$$

A tedious (and so omitted) case analysis shows that if $\mu(G)=2$, then $\operatorname{id}(G) \in T_{1} \cup T_{2} \cup T_{3} \cup T_{4}$, where

$$
\begin{aligned}
& T_{1}=\{1 / 4,3 / 16,7 / 32\}, \\
& T_{2}=\left\{2^{-2}+2^{-A}: A \geq 3\right\}, \\
& T_{3}=\left\{2^{-2}+2^{-A}+2^{-B}: 3 \leq A \leq B\right\},
\end{aligned}
$$

and

$$
T_{4}=\left\{2^{-2}+2^{-A}+2^{-B}+2^{-C}: 3 \leq A \leq B \leq C \leq A+B-2\right\} .
$$

We note that existence and uniqueness of independence density for infinite graphs generalizes to many other graph parameters. A class $X$ of graphs closed under isomorphism is hereditary if it is closed under taking induced subgraphs. For example, the class of independent sets, the class of cliques, $k$-colourable graphs with $k \geq 2$ fixed, perfect graphs, or $H$-free graphs with $H$ a fixed graph are all hereditary. For $X$ a hereditary class of graphs, define $X d(G)$ to be the proportion of subsets which induce a graph in $X$, and extend this definition to countable graphs via chains. It is not hard to see that $X d(G)$ exists and is unique for all countable graphs. We will consider the $X d(G)$ parameters for various classes in the sequel. 


\section{Chromatic densities}

We now consider the chromatic density of a graph. For a finite graph $G$ of order $n$, let $c(G, x)$ denote the chromatic polynomial (or chromial) of $G$, and consider $c(G, n)$ the number of proper $n$-colourings of $G$. It is evident that $c(G, n) \leq n^{n}$ with equality if and only if $G$ has no edges. We define the chromatic density of $G$ by

$$
\operatorname{cd}(G)=\frac{c(G, n)}{n^{n}}
$$

The parameter $\operatorname{cd}(G)$ may be viewed as the probability that a random mapping from $V(G)$ to $\{1,2, \ldots, n\}$ is a proper $n$-colouring (which is a special case of homomorphism density; see [13]).

Given a chain of finite graphs $\mathcal{C}=\left(G_{n}: n \geq 0\right)$ with limit $G$, we define the chromatic density of $G$ relative to the chain $\mathcal{C}$, written $\operatorname{cd}(G, \mathcal{C})$, to be $\lim _{n \rightarrow \infty} \operatorname{cd}\left(G_{n}\right)$, assuming the limit exists. Other notions of limits of chromials (unrelated to our definition) were studied in $[1,17]$.

Regardless of the chain used, the infinite complete graph and its complement, have chromatic densities 0 and 1 , respectively. A tree of order $n$ has $n(n-1)^{n-1}$ many proper $n$-colourings, and hence, has chromatic density $\left(\frac{n-1}{n}\right)^{n-1}$. If we consider a chain $\mathcal{C}$ of trees $T_{n}$ of order $n$ with limit $T$, then

$$
\operatorname{cd}(T, \mathcal{C})=\lim _{n \rightarrow \infty}\left(\frac{n-1}{n}\right)^{n-1}=\frac{1}{e}
$$

(and hence, $\operatorname{cd}(T, \mathcal{C})$ is irrational). Similarly, the limit of a chain of $k$-trees (see [5] for a definition) has chromatic density $(1 / e)^{k}$. However, other chains in a tree may have different limits. For example, index the vertices of the ray by the natural numbers, and define $G_{n}$ inductively as follows. Let $G_{0}$ be the subgraph induced by $\{0\}$. Assume that $G_{n}$ is defined, finite, and $V\left(G_{n}\right)$ contains $\{0,1, \ldots, n\}$. Define $G_{n+1}$ to consist of the smallest order path in the ray containing $V\left(G_{n}\right) \cup\{n+1\}$, along with $2^{\left|V\left(G_{n}\right)\right|}$-many isolated nodes (chosen from vertices with an index greater than $n+3$ ). Then the ray is the limit of $\left(G_{n}: n \geq 0\right)$ and has chromatic density 1 relative to this chain.

Define the probability space $G(\mathbb{N}, p)$ to be graphs whose vertex set is the set of all positive integers, and each distinct pair of integers is joined independently with probability $p \in(0,1)$. We will call this space the infinite random graph. Erdős and Rényi [10] discovered the following theorem.

Theorem 3.1. For $p \in(0,1)$, with probability 1 the graph $G(\mathbb{N}, p)$ is unique up to isomorphism. 
Define a graph to be existentially closed or e.c. if all finite disjoint sets of vertices $A$ and $B$ (one of which may be empty), there is a vertex $z$ joined to all of $A$ and to no vertex of $B$. The proof of Theorem 3.1 follows by proving that with probability $1, G(\mathbb{N}, p)$ is e.c., and then proving that any two e.c. graphs are isomorphic. For more on e.c. graphs, see [2, 9].

The unique isomorphism type of the infinite random graph is written $R$. We exploit the following explicit representation of $R$ as a limit graph. For a graph $G$, for each non-empty subset $S$ of $V(G)$, add a new vertex joined to $S$ and to no other vertices. The resulting graph contains $G$ as an induced subgraph. We may iterate this process, to form a chain of graphs $\left(G_{n}: n \geq 0\right)$ whose limit is e.c., and so is isomorphic to $R$. (Choose $n$ large enough such that $G_{n}$ contains both $A$ and $B$. A vertex joined to $A$ and not $B$ may be found in $G_{n+1}$.)

Our main theorem of this final section demonstrates that chromatic density depends heavily on the chain used. Unlike the results from Theorem 2.4, $R$ realizes all real numbers in $[0,1]$ as a chromatic density.

Theorem 3.2. For all $r \in[0,1]$, there is a chain $\mathcal{C}_{r}=\left(R_{n}: n \geq 0\right)$ of finite graphs whose limit is $R$, with $\operatorname{cd}\left(R, \mathcal{C}_{r}\right)=r$.

In order to prove the result, we first prove a sequence of three lemmas. A vertex is universal if it is joined to all others.

Lemma 3.3. Given a graph $G$ of order $n$, let $G+$ be the graph formed by adding a universal vertex to $G$. If $\operatorname{cd}(G)=x$, then

$$
\operatorname{cd}(G+)=(1+O(1 / n))(x / e)
$$

Proof. We have that

$$
\begin{aligned}
\operatorname{cd}(G+) & =\frac{(n+1) c(G, n)}{(n+1)^{n+1}} \\
& =\frac{c(G, n)}{n^{n}}(1+1 / n)^{-n} \\
& =x \exp \left(1 / n+O\left(1 / n^{2}\right)\right)^{-n} \\
& =(x / e) \exp (-O(1 / n)) \\
& =(1+O(1 / n))(x / e) .
\end{aligned}
$$

In particular, by adding $k$ universal vertices in succession, we divide the chromatic density by a factor of $e^{k}$ (with vanishing error term). Hence, we can make the chromatic density as close to 0 as we like. 
Lemma 3.4. Form $G(-m)$ by adding an independent set of cardinality $m$ to a finite graph $G$ of order $n$. If $m \gg n$ (with both $m$ and $n$ tending to infinity), then $\operatorname{cd}(G(-m))=1+o(1)$.

Proof. We have that

$$
\begin{aligned}
\operatorname{cd}(G(-m)) & =\frac{(m+n)^{m} c(G(-m), n+m)}{(n+m)^{n+m}} \\
& \geq \frac{c\left(K_{n}, n+m\right)}{(m+n)^{n}} \\
& =\frac{(m+n) ! / m !}{(m+n)^{n}} .
\end{aligned}
$$

Using Stirling's formula (that is, $\left.n !=(1+o(1)) \sqrt{2 \pi n}\left(\frac{n}{e}\right)^{n}\right)$ twice, (3.1) is asymptotically equal to

$$
(\sqrt{1+n / m}) e^{-n}\left(\frac{m+n}{m}\right)^{m}=(1+o(1)) e^{-n}\left(1+\frac{n}{m}\right)^{m},
$$

which in turn is asymptotically equal to 1 .

Lemma 3.5. Adding one edge $e=u v$ to a finite graph $G$ of order $n$ does not change the chromatic density by more than $1 / n$.

Proof. By the deletion-contraction rule for the chromial,

$$
\begin{aligned}
\left|\frac{c(G, n)}{n^{n}}-\frac{c(G+u v, n)}{n^{n}}\right| & =\frac{c(G / u v, n)}{n^{n}} \\
& \leq \frac{n^{n-1}}{n^{n}} \\
& =\frac{1}{n} .
\end{aligned}
$$

Proof of Theorem 3.2. Fix $r \in[0,1)$. Let $R_{0}=K_{1}$, and assume $R_{n}$ is defined for $n \geq 0$. First, for each subset $S$ of vertices of $R_{n}$ add a vertex $x_{S}$ joined only to the vertices of $S$; call the resulting graph $G_{n}$. Doing this for all $S$ and for all $n$ will ensure that the resulting limit graph is e.c., and so is isomorphic to $R$. We note that the graph $G_{n}$ is an auxiliary step in the construction of $R_{n+1}$.

Now $\operatorname{cd}\left(G_{n}\right)=y$ for some rational $y$ in $(0,1]$. The idea for the remainder of the proof is to add an independent set to $G_{n}$ yielding a graph with chromatic density above $r$, and then add edges to bring the chromatic density to 
within distance $1 / n$ of $r$. Now, first form $G_{n}(-m)$ from $G_{n}$ for some large $m$ so that $\operatorname{cd}\left(G_{n}(-m)\right) \geq r$ (using Lemma 3.4). By Lemma 3.5 adding an edge to $G_{n}(-m)$ decreases the chromatic density by at most $1 / n$ (in fact, since $n<\left|V\left(G_{n}\right)\right|$, the chromatic density decreases by at most $\left.1 /\left|V\left(G_{n}\right)\right|<1 / n\right)$. Lemma 3.3 implies that adding all edges will decrease the chromatic density by dividing by $e^{m}$, which we may make as close to 0 as we like by making $m$ large enough. Hence, we may obtain a graph $R_{n+1}$ by adding some (deterministic but unspecified) number of edges to $G_{n}(-m)$ so that

$$
\left|\operatorname{cd}\left(R_{n+1}\right)-r\right| \leq 1 / n .
$$

The limit of the chain $\mathcal{C}_{r}=\left(R_{n}: n \geq 1\right)$ is e.c. as we already made all extensions of $R_{n}$ in the graph $G_{n}$, and $R_{n}$ is an induced subgraph of $R_{n+1}$. Hence, the limit of the chain is indeed the infinite random graph $R$ and $\operatorname{cd}\left(R, \mathcal{C}_{r}\right)=r$.

In the case $r=1$, proceed similarly as before. However, to form $R_{n+1}$ in this case, take $m$ sufficiently large that $\operatorname{cd}\left(G_{n}(-m)\right)>1-1 / n$.

\section{Acknowledgements}

The authors gratefully acknowledge support from NSERC, MITACS, Ryerson University, and West Virginia University. We thank the anonymous referees for helpful comments which improved the paper.

\section{References}

[1] N. Biggs, Algebraic Graph Theory, Second Edition, Cambridge University Press, Cambridge, 1993. MR1271140

[2] A. Bonato, A Course on the Web Graph, American Mathematical Society Graduate Studies Series in Mathematics, Providence, Rhode Island, 2008.

[3] A. Bonato, G. Hahn, C. Wang, The cop density of a graph, Contributions to Discrete Mathematics 2 (2007) 133-144. MR2358266

[4] A. Bonato, M. E. Messinger, P. Prałat, Fighting constrained fires in graphs, accepted to Theoretical Computer Science, 16 pp.

[5] A. Brandstädt, V. B. Le, J. P. Spinrad, Graph Classes, a Survey, SIAM Monographs on Discrete Mathematics and Applications, 1999. MR1686154 
[6] J. I. Brown, K. Dilcher, R. J. Nowakowski, Roots of independence polynomials of well covered graphs, J. Algebraic Combin. 11 (2000) 197-210. MR1771611

[7] J. I. Brown, C. A. Hickman, R. J. Nowakowski, On the location of roots of independence polynomials, J. Algebraic Combin. 19 (2004) 273-282. MR2071474

[8] M. Chudnovsky, P. Seymour, The roots of the independence polynomial of a claw-free graph, J. Combin. Theory Ser. B 97 (2007) 350-357. MR2305888

[9] R. Diestel, Graph Theory, Springer-Verlag, New York, 2000. MR1743598

[10] P. Erdős, A. Rényi, Asymmetric graphs, Acta Mathematica Academiae Scientiarum Hungaricae 14 (1963) 295-315. MR0156334

[11] Z. Füredi, The number of maximal independent sets in connected graphs, J. Graph Theory 11 (1987) 463-470. MR0917193

[12] V. Linek, Bipartite graphs can have any number of independent sets, Discrete Mathematics 76 (1982) 131-136. MR1005417

[13] L. Lovász, B. Szegedy, Limits of dense graph sequences, J. Combin. Theory Ser. B 96 (2006) 933-957. MR2274085

[14] R. E. Merrifield, H. E. Simmons, Topological Methods in Chemistry, John Wiley \& Sons, New York, 1989.

[15] A. S. Pedersen, P. D. Vestergaard, Bounds on the number of vertex independent sets in a graph, Taiwanese J. Math. 10 (2006) 1575-1587. MR2275147

[16] P. Prałat, Graphs with average degree smaller than 30/11 burn slowly, preprint.

[17] A. Procacci, A. B. Scoppola, B. V. Gerasimov, Potts model on infinite graphs and the limit of chromatic polynomials, Communications in Mathematical Physics 235 (2003) 215-231. MR1969726

[18] B. E. Sagan, A note on independent sets in trees, SIAM J. Discrete Math. 1 (1988) 105-108. MR0936612 


\author{
ANTHONY BONATO \\ Department of Mathematics \\ RYERSON UNIVERSITY \\ Toronto, ON, M5B 2K3 \\ CANADA \\ E-mail address: abonato@ryerson.ca \\ JASON I. BROWN \\ Department of Mathematics and Statistics \\ DALHOUSIE UNIVERSITY \\ HaLIFAX, NS, B3H 3J5 \\ CANAdA \\ E-mail address: brown@mathstat.dal.ca \\ Graeme Kemkes \\ Department of Mathematics \\ RYERSON UNIVERSITY \\ Toronto, ON, M5B 2K3 \\ CAnada \\ E-mail address: gdkemkes@ryerson.ca \\ PaWel Prąat \\ Department of Mathematics \\ RYERSON UNIVERSITY \\ Toronto, ON, M5B 2K3 \\ CANADA \\ E-mail address: pralat@math.wvu.edu
}

Received December 13, 2010 